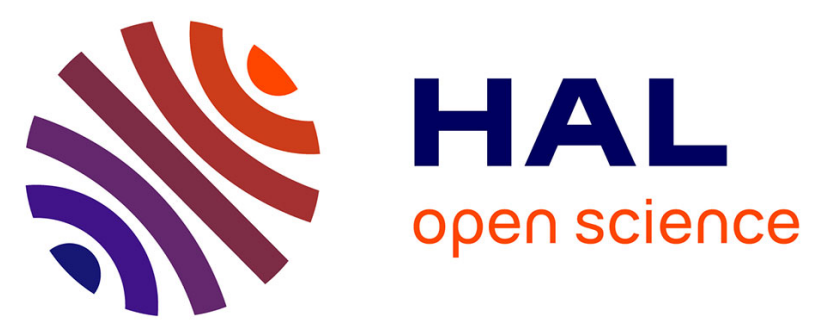

\title{
A traffic aware joint CQI feedback and scheduling scheme for multichannel downlink systems in TDD feedback mode
}

Apostolos Destounis, Mohamad Assaad, Mérouane Debbah, Bessem Sayadi

\section{- To cite this version:}

Apostolos Destounis, Mohamad Assaad, Mérouane Debbah, Bessem Sayadi. A traffic aware joint CQI feedback and scheduling scheme for multichannel downlink systems in TDD feedback mode. 2013 IEEE 24th Annual International Symposium on Personal, Indoor, and Mobile Radio Communications (PIMRC), Sep 2013, London, United Kingdom. pp.1523 - 1527, 10.1109/PIMRC.2013.6666383 • hal00927744

\section{HAL Id: hal-00927744}

https://hal-centralesupelec.archives-ouvertes.fr/hal-00927744

Submitted on 13 Jan 2014

HAL is a multi-disciplinary open access archive for the deposit and dissemination of scientific research documents, whether they are published or not. The documents may come from teaching and research institutions in France or abroad, or from public or private research centers.
L'archive ouverte pluridisciplinaire HAL, est destinée au dépôt et à la diffusion de documents scientifiques de niveau recherche, publiés ou non, émanant des établissements d'enseignement et de recherche français ou étrangers, des laboratoires publics ou privés. 


\title{
A traffic aware joint CQI feedback and scheduling scheme for multichannel downlink systems in TDD feedback mode
}

\author{
Apostolos Destounis*, Mohamad Assaad ${ }^{\ddagger}$, Mérouane Debbah ${ }^{\ddagger}$, Bessem Sayadi* \\ *Alcatel-Lucent Bell Labs France, \{apostolos.destounis, bessem.sayadi\}@alcatel-lucent.com \\ ${ }^{\ddagger}$ SUPÉLEC, France, \{mohamad.assaad, merouane.debbah\}@ supelec.fr
}

\begin{abstract}
In this work we study the problem of channel state feedback and user scheduling in a single cell downlink wireless network employing multiple orthogonal parallel channels. The aspect of the system we are focusing on is stability. For user scheduling for stability as a performance measure, both the queue and channel states need to be known by the base station. However channel states can be known only via feedback from the receivers. In order to collect CQI feedback from each user at one channel, a fraction of the available time for transmission is used. This means that the time left to transmit is getting smaller. We present a joint feedback and scheduling algorithm which can guarantee an expansion of the stability region with respect to prior works. We also provide expressions regarding the distribution of the time needed to be devoted for feedback at each channel in some special cases. The proposed algorithm does not need knowledge of the statistics of the channels and traffic patterns. Simulations illustrate the operation of the proposed scheme.
\end{abstract}

\section{INTRODUCTION}

User scheduling has emerged as an attractive solution to improve the performance of wireless networks by allocating the resources (timeslots, frequencies) to the users depending on their channel states. On the other hand, since each user in the network is associated with an incoming traffic process, stability is among the first-order desirable properties (performance metrics) of a scheduler. It roughly means that the mean of all the queue lengths (and consequently delays experienced by the users) in the network is finite. It was shown that MaxWeight types of scheduling policies are throughput optimal, i.e. stabilizing the system if it can indeed be stabilized [1], [2]. However, these works assume that the realizations of the channel processes are known to the scheduler at each time slot, which can only be done by feedback from the receivers. The cost, in terms of resources, needed to acquire the instantaneous channel processes is neglected in these works.

This problem of limited feedback in wireless systems has recently been the subject of quite intensive research, however in most works (e.g. see [3] and references therein, also [4]) the focus is on maximizing the total throughput, not taking queueing behaviour into account. Regarding the effect of feedback on stability performance, the authors in [5] study the problem of deciding which subset of users to collect feedback from, while the authors in [6] investigate the achievable stability region in a multichannel system with infrequent channel measurements. In these works channel statistics are assumed known . Moreover, in [7], a CSMA-based scheme is presented for channel state feedback and in [8] the authors devise a feedback scheme for a multiuser MIMO downlink employing orthonormal beamforming. In these cases however the authors do not take into account the fact that the base station must wait for some time in the slot before the feedback can be used. Assuming channel statistics are known, the authors in [9] propose a heuristic feedback scheme with two feedback slots based on the idea of maximum quantile scheduling. Furthermore, in [10] it is shown that for a system of $L$ carriers with FDD mode for feedback, the base station needs to acquire at least $\Theta(L)$ channel realizations each time slot to obtain very close to the biggest achievable stability region. In [11], a TDD mode of feedback is used: the base station manually requests the users to feed back their channel states but each procedure is centralized and takes up a portion of the time slot. Based on optimal stopping theory and assuming that the distributions of the channel gains are known to the base station, the authors derive the general properties of the centralized optimal probing policy and completely characterize it in some special cases. Finally, for the same model, the authors in [12], [13] have recently propose a simple feedback scheme for a single channel system. This scheme requires no knowledge of channel and traffic statistics and is shown to guarantee greater stability region than a scheme where all channels are probed. In multi-carrier systems, the probing problem is more challenging since a user may be scheduled on a subset of channels and therefore each user needs to feed back the channel state informations CSIs of a subset (as small as possible) of its channels. Applying directly the aforementioned schemes to multi-carrier systems may not result in a good stability region because the number of users feeding back on each channel might be still big. This poses more of a problem as the number of users in the cell increases. In [14] the authors addressed the same problem we consider here and introduced a randomized scheme based on this work in which a user feeds back at every channel with some properly defined probability. However the policy obtained therein was based on loose bounds and the analytical results are valid for the case where there is enough time for everyone to feed back.

In this paper, we focus on the downlink of a multichannel single cell system with feedback in TDD mode. We propose a scheme where a threshold for the achievable rate of the channel is adjusted by the base station according to the queue lengths 
of the users and then users with rate above the threshold feed back in this channel (in a way similar to [13]). However, at every channel, the base station can stop the process and transmit anytime if no improvement is going to happen by letting more users feeding back. The analysis and main result is valid for any number of users and feedback cost.

The rest of the paper is organized as follows: In Section II we present the system model. In Section III, we describe the proposed feedback and scheduling scheme and provide some insight on its behaviour in terms of number of users feeding back. In Section IV, we present simulation results and a discussion on the performance of the proposed scheme and Section V concludes the paper.

\section{SYSTEM MODEL AND BASIC NOTIONS}

We consider a single cell multi-carrier system where a base station serves $K$ users using $N$ channels, assumed to be randomly time varying, i.i.d. across time. This can model the case of OFDMA downlink schemes with $N$ carriers or the case where the base station is equipped with $N$ antennas and orthonormal beamforming is used (in the latter case a "channel" is a beamforming vector). Time is slotted. Let $R_{k n}(t)$ be the achievable rate for user $k$ at channel $n$ at timeslot $t$ (in bits per timeslot duration). This rate is assumed to belong to a set of finite values, $\left\{r_{1}, . ., r_{L}\right\}, r_{1}=0, r_{l+1}>r_{l}$, which is the case in practical systems, as a finite number of modulation and coding schemes is used. Also the rates are independent from each other and across users, but not necessarily identically distributed. Each user $i \in\{1, \ldots, K\}$ is associated with a randomly incoming traffic process with mean rate $\lambda_{i}$. Incoming traffic processes are i.i.d. across time, independent across users and independent with respect to the channel processes.For the MAC layer, the base station maintains a different queue for each user, whose queue length at time slot $t$ is denoted $Q_{i}(t)$.

Central in our case is the notion of stability of the system. We say that the system is (strongly) stable if for every queue $i$ it holds $\lim _{T \rightarrow \infty} \sup \frac{1}{T} \sum_{t=1}^{T} \mathbb{E}\left\{Q_{i}(t)\right\}<+\infty$. This implies that the process of queue lengths converges to an ergodic distribution and that the queues (therefore delays) for each user will be finite.

Definition 1 (Stability Region). The stability region $\Lambda$ of an algorithm is defined as the set of vectors of the arrival rates for which the system is stable under this algorithm.

Define now the weight of user $k$ at channel $n$ as $W_{k n}(t)=$ $Q_{t}(t) R_{k n}(t)$. If all channel realizations are known, the MaxWeight scheduler, where at each channel $n$ the user with the maximum weight is scheduled achieves the biggest stability region possible [2]. However, we consider feedback in TDD mode and the slot divided in minislots of duration $\beta T_{s}$. At each minislot, at each channel the base station can either request a user to feed back on this subcarrier, broadcast information or let users feed back in a decentralized way. In channel $n$, let $M_{n}(t)$ be the number of minislots used by the feedback procedure at time slot $t$; then, if user $k^{*}(n)$ is scheduled, it will receive $\left(1-\beta M_{n}(t)\right) R_{k^{*}(n) n}(t)$ bits at timeslot $t$. Define $Z_{k n}(t)$ the scheduling decision at time slot $t$ (i.e. $Z_{k n}(t)=1$ if user $k$ is scheduled on channel $n$ at time slot $t$ and zero otherwise). As mentioned in the Introduction, we will compare our scheme with the scheme of [13] applied in multiple carriers (will be referred to as "SSF" in the rest of the paper, standing for "Selective Scheduling and Feedback" [12], [13]). Variables with a tilde will be the quantities corresponding to the proposed scheme, while variables denoted with normal letters will correspond to the SSF scheme. Note then that $Z_{k n}(t)$ is the same schedule as MaxWeight scheduling when all the channels were known [13].

Define the following quantities under the two scheduling and feedback schemes:

$$
\begin{aligned}
& f(\mathbf{Q}(t))= \\
& \mathbb{E}\left\{\sum_{n=1}^{N}\left[1-\beta M_{n}(t)\right]^{+} \sum_{i=1}^{K} Q_{i}(t) R_{i n}(t) Z_{i n}(t) \mid \mathbf{Q}(t)\right\} \\
& \tilde{f}(\mathbf{Q}(t))= \\
& \mathbb{E}\left\{\sum_{n=1}^{N}\left[1-\beta \tilde{M}_{n}(t)\right]^{+} \sum_{i=1}^{K} Q_{i}(t) R_{i n}(t) \tilde{Z}_{i n}(t) \mid \mathbf{Q}(t)\right\} .
\end{aligned}
$$

Note that these quantities correspond to negative part of the drift of the quadratic Lyapunov function under the aforementioned schemes. Then, the following holds (see [15], also e.g. [13], [10]):

Theorem 2. If there exists an $\epsilon>0$ such that for every queue length vector $\mathbf{Q}(t)$ it holds

$$
\frac{\tilde{f}(\mathbf{Q}(t))}{f(\mathbf{Q}(t))} \geq 1+\epsilon,
$$

then $\tilde{\Lambda} \supseteq(1+\epsilon) \Lambda$.

Since at most one user can be scheduled on a channel, $Z_{k n}(t)=1$ only for the user with the maximum weight at channel $n$.

Unless stated otherwise, all expectations in the remainder of the paper are taken over the stationary distribution of the channel states and the decisions taken.

\section{FEEDBACK AND SCHEDULING SCHEME}

\section{A. Proposed Scheme}

We assume that at the beginning of each timeslot the base station broadcasts a pilot signal of negligible duration, so that the users can know their current channel states. Denote $\mathcal{U}_{n}(m, t)$ the set of users that have fed back at control slot $m$ of timeslot $\mathrm{t}$. The proposed algorithm actually considers every channel in isolation and consists in the following steps for every time slot $t$ at each channel $n$ :

1) The base station requests the CQI of the user with the biggest queue length, $k^{*}$.

2) If after receiving feedback in the first minislot it holds that $\frac{1-\beta}{1-3 \beta} \frac{Q_{k^{*}}(t)}{Q_{k}(t)} R_{k^{*} n}(t) \geq r_{L}, \forall k \neq k^{*}$, the base 
station transmits at user $k^{*}$ at its achievable rate for the rest of the timeslot (and so the algorithm terminates). Otherwise, it broadcasts $R_{t h r, n}(t):=R_{k^{*} n}(t)$ during the second feedback minislot.

3) For each minislot $m>2$, at the beginning the base station chooses $k^{*}=\arg \max _{k \in \mathcal{U}_{n}(m, t)}\left\{R_{k n}(t) Q_{k}(t)\right\}$. If it holds that $\frac{1-m \beta}{1-(m+1) \beta} \frac{Q_{k^{*}}(t)}{Q_{k}(t)} R_{k^{*} n}(t) \geq r_{L}, \forall k \notin$ $\mathcal{U}_{n}(m, t)$ then the base station transmits to user $r^{*}$ and the algorithm terminates. Otherwise, user $i \notin \mathcal{U}_{n}(m, t)$ with rate $R_{i n}(t)>R_{t h r, n}$ feeds back according to a decentralized rule.

4) The algorithm stops when there is no user to feed back on channel $n$ (that is all remaining users not yet fed back have worse channel state than the one broadcasted) or when $m=\left\lfloor\frac{1}{\beta}\right\rfloor$. The latter means that this is the last minislot; in this case, the base station transmits to the user with the maximum weight among the ones that fed back.

The main idea behind the algorithm is to transmit when there is no possibility that receiving further feedback will increase the weight of the user scheduled in the channel, thus increasing (2). For example, if the user with the maximum queue length has the maximum possible rate allowed by the standard on channel $n$, then it is useless to do the procedure of feedback, since it is the user with the maximum weight in this channel. Formally we can show the following:

Proposition 3. Under the algorithm described in this section, $\tilde{\Lambda} \supset \Lambda$, where $\Lambda$ is the stability region when the algorithm in [13] is used.

Proof. We consider the beginning of minislot $m$ at channel $n$ at timeslot $t$ and denote $k_{n}^{*}(m, t)=$ $\arg \max _{k \in \mathcal{U}_{n}(m, t)}\left\{R_{k n}(t) Q_{k}(t)\right\}$, that is the user with the maximum weight at this channel so far. If $m>2$, then if user $i \notin \mathcal{U}_{n}(m, t)$ feeds back, the maximum weight of the channel in minislot $m+1$ will increase if $(1+\beta(m+1)) R_{\text {in }}(t) Q_{i}(t)>$ $(1-\beta m) \max _{k \in \mathcal{U}_{n}(m, t)}\left\{R_{k n}(t) Q_{k}(t)\right\}$. This implies that, since the queue lengths vector is known to the base station, the weight in this channel gets bigger if $i \notin \mathcal{U}_{n}(m, t)$ feeds back at minislot $m$ if

$$
\begin{aligned}
R_{i n}(t) & >\hat{R}_{i n}(m, t) \\
& :=\frac{1-\beta m}{1-\beta(m+1)} \frac{\max _{k \in \mathcal{U}_{n}(m, t)}\left\{R_{k n}(t) Q_{k}(t)\right\}}{Q_{i}(t)} .
\end{aligned}
$$

Consider now the case where we have

$$
\hat{R}_{i n}(m, t) \geq r_{L}, \forall i \notin \mathcal{U}_{n}(m, t)
$$

Since $\frac{1-\beta m}{1-\beta(m+1)}$ is an increasing sequence in $m, \hat{R}_{i n}(m+$ $1, t)>\hat{R}_{i n}(m, t)>r_{L}, \forall i \notin \mathcal{U}_{n}(m, t)$. This analysis implies that if (5) holds in the beginning of minislot $m$ then the weight of the user scheduled at channel $n$ will not increase any further. Similar analysis holds for $m=1$ as well, taking though into account that if the base station decides not to transmit and at least one user is above the threshold, then it can transmit again after minislot $m=3$ the earliest due to the second minislot used for broadcasting (thus the denominator of Step 2 in the algorithm).

The above implies that, given any (possibly randomized) rule for the decentralized feedback scheme, for any realization of this rule under any realization of the channel states and any fixed queue length vector we have that if $R_{k^{*}(n) n(t)}<r_{L}$, it holds $\quad\left[1-\beta \tilde{M}_{n}(t)\right]^{+} \sum_{i=1}^{K} Q_{i}(t) R_{i n}(t) \tilde{Z}_{i n} \geq$ $\left[1-\beta M_{n}(t)\right]^{+} \sum_{i=1}^{K} Q_{i}(t) R_{i n}(t) Z_{i n}$ with probability 1 . In the case where the $R_{k^{*}(n) n(t)}=r_{L}$, the user with the maximum queue length is the user with the maximum weight already. This user is scheduled right after the first minislot in our algorithm while under SSF the second minislot is also used for the broadcasting of this rate, so the weight under our algorithm in this case is stricly bigger than SSF with probability one. This analysis implies that for every channel $n=1, \ldots, N$

$$
\begin{gathered}
\mathbb{E}\left\{\left[1-\beta \tilde{M}_{n}(t)\right]^{+} \sum_{i=1}^{K} Q_{i}(t) R_{i n}(t) \tilde{Z}_{i n} \mid \mathbf{Q}(t)\right\}> \\
\mathbb{E}\left\{\left[1-\beta M_{n}(t)\right]^{+} \sum_{i=1}^{K} Q_{i}(t) R_{i n}(t) Z_{i n} \mid \mathbf{Q}(t)\right\} .
\end{gathered}
$$

Summing over all channels and using the fact that they are independent we get $\tilde{f}(\mathbf{Q}(t))>f(\mathbf{Q}(t))$, and combining this with Theorem 2 completes the proof.

A further issue is how exactly the users that have better rate than the broadcasted one can be coordinated to feed back. This can be done for example if the base station ranks the users and communicates this ranking with them (i.e. it can be a ranking according to their IDs, communicated at the beginning of the systems' operation), and divides the portion of the second minislot that remains after the threshold broadcast among the users (in a TDMA manner in each channel). There when it $\mathrm{s}$ the turn of each user, they can send a signal if their rate at the channel is above the threshold and send nothing otherwise. In any case, the number of minislots used for the feedback phase for the SSF algorithm does not depend on the way the users above the threshold feed back, our algorithm outperforms SSF under any user ordering scheme. However, in principle the actual ordering scheme will affect the stability region of our algorithm. For the threshold broadcast step to make sense, we must have $\beta<1 / 3$ and $K>2$ users.

\section{B. Analysis of the time spent for feedback for i.i.d. channels}

Here we will provide some mathematical analysis on the number of minislots taken up by our proposed policy. In order to simplify the model, we will assume that all channels are identically distributed with $\mathbb{P}\left\{R_{k n}(t)=r_{l}\right\}=q_{l}$. In addition, we will assume that the users feed back according to a ranking based on the queue lengths. This can be implemented as follows: The base station can broadcast a ranking of the users according to the queue lengths with the user with the highest queue length first at the beginning of the timeslot (e.g.at the beginning of the first minislot and then in the 
remaining time of this minislot the first user in the ranking feeds back in all channels). Then, the procedure described in the previous subsection for determining the sequence at which users will feed back is followed. Note that, since we are actually interested in maximizing the quantity (2) and the channels are iid, this method will give the biggest stability region (biggest value of (2)) over any feedback sequence under the proposed scheme.

Given the stopping condition at each minislot and the above mentioned feedback scheme, we can further see that the expected number of minislots used is the biggest when the queue lengths are equal since it leads to $\frac{Q_{k^{*}}(t)}{Q_{k}(t)}=1$ in the stopping condition. Therefore we will examine this setting in order to obtain a worst case analysis of the scheme. Since all queues are equal, without loss of generality, ranking will be assumed to be according to the user IDs in ascending order (i.e. user 1 feeds back first etc.). Denote $\tilde{p}_{n}(m)$ the probability that exactly $m$ minislots are used at carrier $n$ under our scheme and $p_{n}(m)$ the corresponding quantity for the SSF algorithm. Note that when $m>\left\lfloor\frac{1}{\beta}\right\rfloor$, more time than the duration of the timeslot needs to be used. So, eventually the base station does not transmit at all in the slot (this goes for the SSF algorithm as our proposed one stops at most after the minislot just before the last that can fit in the timeslot duration). Also denote $F_{l}=\mathbb{P}\left\{R_{k n} \geq r_{l}\right\}=\sum_{l=l}^{L} q_{l}$, so $F_{L+1}=0$. For the SSF algorithm, the number of minislots needed is the number of users out of the remaining $K-1$ that have rates over the threshold plus the two minislots in the beginning, so we have for

$$
p_{n}(m)=\sum_{l=1}^{L} q_{l}\left(\begin{array}{c}
K-1 \\
m-2
\end{array}\right) F_{l+1}^{m-2}\left(1-F_{l+1}\right)^{K-m+1}, m \geq 2
$$

and zero for $\mathrm{m}=1$.

For the proposed scheme, note that we have for $m=1$

$$
\tilde{p}_{n}(1)=\sum_{l=1}^{L} q_{l} \mathbb{1}_{\left\{r_{l} \geq \frac{1-3 \beta}{1-\beta} r_{L}\right\}} .
$$

For $m=2$, it corresponds to the case when the stopping condition is not fulfilled after the user with the maximum queue lengths feeds back but no user among the remaining $K-1$ has greater rate, thus we have

$$
\tilde{p}_{n}(2)=\sum_{l=1}^{L} q_{l} \mathbb{1}_{\left\{r_{l}<\frac{1-3 \beta}{1-\beta} r_{L}\right\}}\left(1-F_{l+1}\right)^{K-1} .
$$

For $m=3$, the corresponding event, given the threshold rate (the rate of the user with the maximum queue length) is that the stopping condition did not hold for the first minislot and that either only one of the $K-1$ users has rate above the threshold or this happens for more than one user but the stopping condition holds after a user feeds back in this minislot. Replacing the probabilities we get

$$
\begin{aligned}
\tilde{p}_{n}(3)= & \sum_{l=1}^{L} q_{l} \mathbb{1}_{\left\{r_{l}<\frac{1-3 \beta}{1-\beta} r_{L}\right\}}\left((K-1) F_{l+1}\left(1-F_{l+1}\right)^{K-2}+\right. \\
& \left(1-(K-1) F_{l+1}\left(1-F_{l+1}\right)^{K-2}-\left(1-F_{l+1}\right)^{K-1}\right) \\
& \left.\sum_{l^{\prime}=l+1}^{L} q_{l^{\prime}} 1_{\left\{r_{l^{\prime}}<\frac{1-4 \beta}{1-3 \beta} r_{L}\right\}}\right) .
\end{aligned}
$$

For $m>3$, getting closed form expressions like the above becomes more difficult, since for every $m$ this probability depends on which users have fed back and their channel realizations, thus boiling down to a combinatorial problem. Define for $m>2$ the outcome of the feedback process until and including the $m$-th minislot as

$$
\begin{aligned}
\pi(m)=( & \left(i_{1}(\pi), R_{i_{1}(\pi) n}(t)\right),(0,0),\left(i_{3}(\pi), R_{i_{3}(\pi) n}(t)\right), \\
& \left.\ldots,\left(i_{m}(\pi), i_{m}(\pi)(t)\right)\right) .
\end{aligned}
$$

More specifically $i_{j}(\pi)$ is the user that fed back at minislot $j \leq m$ and $R_{i_{j}(\pi) n}(t)$ the corresponding achievable rate. A realization $\pi(m)=\left(\left(i_{1}, r^{(1)}\right),(0,0), \ldots,\left(i_{j}, r^{(j)}, . .,\left(i_{m}, r^{(m)}\right)\right)\right.$ is possible if the following conditions are met: (i) $r^{(j)}>$ $r^{(1)}, \forall j=3, . ., m,(\mathrm{ii})(1-\beta) r^{(1)}<(1-3 \beta) r_{L}$ (iii) $(1-$ $\beta j) \max \left\{r^{(1)}, r^{(3)}, \ldots, r^{(j-1)}, r^{(j)}\right\}<(1-\beta(1+j)) r_{L}, \forall j=$ $1, . ., m-1$ and (iv) either $m-1$ exactly user have rates above the threshold or $(1-\beta m) \max \left\{r^{(1)}, r^{(3)}, \ldots, r^{(m-1)}, r^{(m)}\right\} \geq$ $(1-\beta(1+m)) r_{L}$. Let $\Pi\left(m,\left(1, r_{l}\right)\right)$ be the set containing all possible realizations of the feedback algorithm lasting exactly $m$ minislots when the user requested to feed back first has rate $r_{l}$. Then we have that for $m>2$ :

$$
\begin{aligned}
\tilde{p}_{n}(m)= & \sum_{l=1}^{L} q_{l} \sum_{\pi \in \Pi\left(m,\left(1, r_{l}\right)\right)} \\
& \prod_{j=3}^{m} \mathbb{P}\left\{R_{i_{j}(\pi) n}(t)=r^{(j)}(\pi)\right\}\left(1-F_{l+1}\right)^{i_{j}(\pi)-i_{j-1}(\pi)} .
\end{aligned}
$$

The above equation comes from the fact that since users are ranked using their IDs, if after user $i_{j-1}$, user $i_{j}$ feeds back, it implies that the users with IDs from $i_{j-1}+1$ till and including $i_{j}-1$ have achievable rates below the broadcasted threshold at channel $n$.

The results in this subsection can be used to numerically obtain an estimate of the mean amount of time needed in each timeslot for the feedback procedure to be executed under our algorithm.

\section{Simulation Results}

In order to illustrate the gains from our proposed feedback and scheduling algorithm, we will consider for convenience a downlink system with $N=15$ channels which identically distributed among them and among users, and i.i.d. in time. The possible rates are derived following the LTE standards. In addition, the traffic processes are Poisson with the same rate for each user and i.i.d. in time. What we are showing 
in this Section, therefore, is stability behaviour on the line $\lambda_{1}=\lambda_{2}=\ldots=\lambda_{K}$ in a system with identical channels for each user. The point where the system is becoming unstable is the point where the total average queue length plotted in the figures that follow starts increasing very steeply. We are comparing the performance of our algorithm with the one in [13] applied directly in multichannel systems.

In Figure 1 we present the simulation results for different numbers of users and $\beta=0.1$. As the number of users grows, the stability region of both algorithms shrinks, and the region under our algorithm is bigger than the region under [13]. However, we can observe that the absolute difference between the two algorithms is very similar for each of the cases shown, which suggests that the absolute difference in the stability regions between the two algorithms does not change much with the number of users. An explanation for this is that the proposed stopping rule does not take at all into account the number of users, so (unless the number of users is so large that there is not enough time for everyone to feed back even in the SSF scheme) the degradation on the stability region of both algorithms is similar.

On the other hand, in Figure 2, we present the results for different values of the fraction of time, $\beta$ for one user to feed back on a channel in a system with 10 users. Unlike the previous case, we observe that relatively small changes to the parameter $\beta$ result to different absolute differences, and more precisely the bigger this parameter is, the bigger is the gain, with respect to the algorithm in [13], of using the proposed algorithm (and again, the stability region of both algorithms shrinks as $\beta$ increases).

\section{CONCLUSIONS}

In this paper we presented a feedback and scheduling algorithm for enlarging the stability region in multichannel systems when a fraction of the timeslot must be taken for each user to feed back. Our algorithm does not require knowledge about statistics of the traffic and the channels and can indeed increase the stability region with respect to the state of the art. Simulation results imply that this increase becomes more significant as feedback becomes more costly. Further work may include modification to take into account other performance measures (e.g. delay) and finding the maximum achievable stability region under this feedback model.

\section{ACKNOWLEDGMENT}

The research of M. Assaad has been supported in part by the Celtic-Plus project SHARING.

\section{REFERENCES}

[1] L. Tassiulas and A. Ephremides, "Stability properties of constrained queueing systems and scheduling policies for maximum throughput in multihop radio networks," IEEE Trans. Autom. Control, vol. 37, no. 12, pp. 1936 -1948, Dec. 1992.

[2] A. L. Stolyar, "Maxweight scheduling in a generalized switch: State space collapse and workload minimization in heavy traffic," Ann. Appl. Probab., vol. 14, no. 1, pp. 1-53, Nov. 2004.

[3] D. Love, R. Heath, V. Lau, D. Gesbert, B. Rao, and M. Andrews, "An overview of limited feedback in wireless communication systems," IEEE J. Sel. Areas Commun., vol. 26, no. 8, pp. $1341-1365$, Oct. 2008.

[4] L. Song, Z. Han, Z. Zhang, and B. Jiao, "Non-cooperative feedback-rate control game for channel state information in wireless networks," IEEE J. Sel. Areas Commun., vol. 30, no. 1, pp. 188 -197, Jan. 2012.

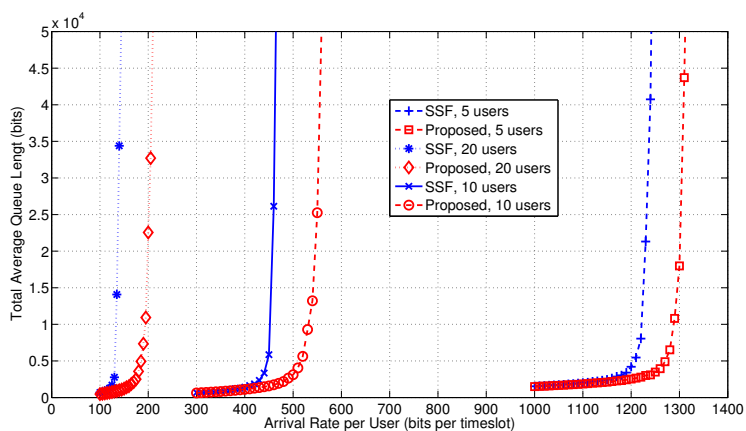

Fig. 1. Average Total Queue Length for Different Mean Arrival Rates for $\beta=0.1$ and different number of users

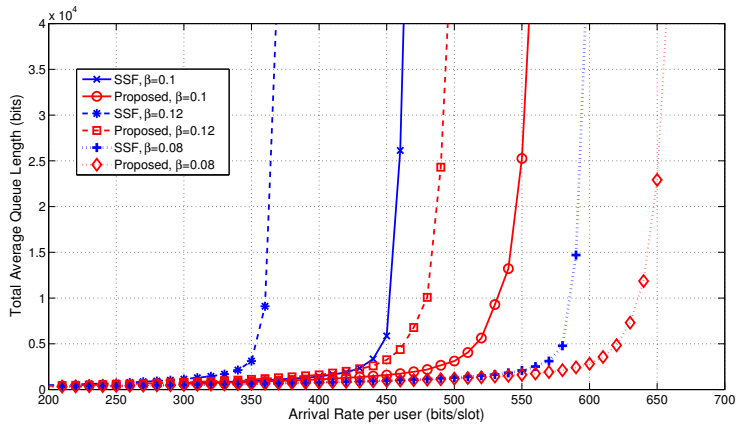

Fig. 2. Average Total Queue Length for Different Mean Arrival Rates for 10 users and different values of $\beta$

[5] A. Gopalan, C. Caramanis, and S. Shakkottai, "On wireless scheduling with partial channel-state information," IEEE Trans. Inf. Theory, vol. 58, no. 1 , pp. $403-420$, Jan. 2012.

[6] K. Kar, X. Luo, and S. Sarkar, "Throughput-optimal scheduling in multichannel access point networks under infrequent channel measurements,' IEEE Trans. Wireless Commun., vol. 7, no. 7, pp. 2619-2629, 2008.

[7] B. Li and A. Eryilmaz, "Distributed channel probing for efficient transmission scheduling over wireless fading channels," in Proc. of IEEE INFOCOM 2012, Mar. 2012, pp. 3026 -3030.

[8] J. Chen and V. K. N. Lau, "Large Deviation Delay Analysis of QueueAware Multi-user MIMO Systems with Multi-timescale Mobile-Driven Feedback," ArXiv e-prints, Nov. 2012.

[9] S. J. Baek and G. de Veciana, "Opportunistic feedback and scheduling to reduce packet delays in heterogeneous wireless systems," IEEE Trans. Vehic. Technol., vol. 61, no. 7, pp. 3282 -3289, Sept. 2012.

[10] M. Ouyang and L. Ying, "On optimal feedback allocation in multichannel wireless downlinks," in Proc. ACM MobiHoc '10, 2010, pp. 241-250.

[11] P. Chaporkar, A. Proutiere, H. Asnani, and A. Karandikar, "Scheduling with limited information in wireless systems," in Proc. ACM Mobihoc '09, 2009, pp. 75-84.

[12] M. Karaca, Y. Sarikaya, O. Ercetin, T. Alpcan, and H. Boche, "Efficient wireless scheduling with limited channel feedback and performance guarantees," in Proc. IEEE PIMRC, Sept. 2012, pp. $106-111$.

[13] - "Joint opportunistic scheduling and selective channel feedback," IEEE Trans. Wireless Commun., accepted

[14] A. Destounis, M. Assaad, M. Debbah, and B. Sayadi, "A randomized probing scheme for increasing the stability region of multicarrier systems," in Proc. IEEE ISIT, 2013 (accepted).

[15] A. Eryilmaz, R. Srikant, and J. R. Perkins, "Stable scheduling policies for fading wireless channels," IEEE/ACM Trans. Netw., vol. 13, no. 2, pp. 411-424, Apr. 2005. 